Accepted for publication in the Bulletin of the Seismological Society of America, $12^{\text {th }}$ July 2020

\title{
Selecting Ground-Motion Models for Site-Specific PSHA: Adaptability vs Applicability
}

\author{
Julian J Bommer and Peter J Stafford \\ Civil \& Environmental Engineering Department, Skempton Building, Imperial College London, \\ South Kensington campus, London SW72AZ, United Kingdom, E: j.bommer@imperial.ac.uk
}

\begin{abstract}
Capturing the center, the body and the range of ground-motion predictions is an indispensable element of site-specific probabilistic seismic hazard analyses (PSHA), for which the logic tree is the ubiquitous tool in current practice. The criteria for selecting the ground-motion models (GMMs) used in such studies have generally been focused on their potential applicability to the region and site for which the PSHA is being conducted. However, except for applications within the few regions with abundant ground-motion databases, it will rarely be the case that GMMs can be identified which are perfectly calibrated to the characteristics of the target study region in terms of source and path properties. A good match between the generic site amplification model within the GMM and the site-specific dynamic response characteristics is equally, if not more, unlikely. Consequently, adjustments are likely to be made to the selected GMMs to render them more applicable to the target region and site. Empirical adjustments for host-to-target region source differences using local recordings are unlikely to be robust unless these have been generated by earthquakes from a wide range of magnitudes. Empirical adjustments for site characteristics are impossible unless there are recordings from the target site. Therefore, the preferred approach makes parametric adjustments to empirical GMMs, isolating each host-to-target difference to map the individual contributions to the epistemic uncertainty. For such an approach to be applied, the emphasis moves from selecting GMMs on the basis of their applicability to focusing on their amenability to being adjusted to the target region and site. An adaptable equation is characterized by well constrained host-region source, path and site characteristics and a functional form in which response spectral accelerations scale with source, path and site characteristics in a manner similar to the scaling implicit in stochastic simulations based on Fourier amplitude spectra.
\end{abstract}




\section{Introduction}

The widely-accepted goal of modern probabilistic seismic hazard analysis (PSHA) is to capture the center, the body, and the range (CBR) of the shaking hazard at a given location (e.g., USNRC, 2018). This objective is reached through capture of the CBR of the technically-defensible interpretations (TDI) of available data, methods and models and their integration into seismic source characterization (SSC) and ground motion characterization (GMC) models. The tool ubiquitously applied towards this objective is the logic tree, in which a node is established for each component of the hazard inputs with weighted branches representing the alternative models or parameter values considered potentially appropriate for that element. The logic-tree branches corresponding to the ground-motion model (GMM) commonly represent alternative models for estimating values of spectral acceleration at the site of interest for specified earthquake scenarios, with the branch weights representing the relative confidence in each model being the best suited to the seismic source and site to which it is being applied. The focus, therefore, has generally been on selecting and weighting GMMs on the basis of their applicability to the region and site under consideration, although this has often been judged on the basis of rather vague notions of regional analogs or tests using local recordings from earthquakes of relatively small magnitude.

In practice, for most site-specific PSHAs performed for critical structures in regions other than the few for which there are abundant databases of ground-motion recordings, it is unlikely that GMMs can be identified that are perfectly applicable to the source and path characteristics of the target region. Moreover, it is also improbable that the generic site amplification factors embedded in a published GMM, conditioned on parameters such as $V_{\mathrm{S} 30}$ (the time-averaged shear-wave velocity in the top $30 \mathrm{~m}$ ) and depth of sediments, will coincide with the dynamic characteristics of the target site. Since the latter, unlike most of the inputs to a PSHA, can be constrained through data collection and modeling without requiring the occurrence of new earthquakes, it is generally considered indispensable to capture the CBR of the local site response. Consequently, it has become common practice to apply adjustments to render published GMMs more applicable to the target region and the target site. We argue that if this is the case, it would be logical to shift the basis for selecting the GMMs for use in PSHA from their potential applicability to the target region and site to their potential for being adapted to the site-specific application.

In order to provide a general framework for the presentation of the proposed shift from selection on the basis of applicability to selection on the basis of adaptability, this paper begins with a brief overview of how ground-motion logic trees have evolved over 35 years of practice. We then discuss in more detail how applicability selection criteria have been defined and the approaches adopted to identify suitable GMMs. Within the same section we also discuss recent models derived to allow for region-specific parameters to be 
included among the explanatory variables, addressing both the advantages and shortcomings of these models.

Following these reviews, we make the case for the procedure of constructing groundmotion logic-trees by harnessing the predictive power and physical constraint of empirical GMMs and adapting these to the conditions of the target region and site through parametric adjustment. We include a description of the various source, path, and site adjustments that can be made, and how these can be applied in a manner that facilitates the tractable inclusion of epistemic uncertainty. The paper then goes on to discuss the features of a GMM that make it more or less amenable to such host-to-target adjustments, including both the characterization of the host-region environment and the functional form of the equation. This is followed by identification of adaptable GMMs for active crustal regions. The paper concludes with a discussion of how adaptability could become an explicit objective in GMM development and the advantages that would be obtained from the generation of adaptable GMMs.

\section{The Evolution of Ground-Motion Logic-Trees}

In the first published illustration of using a logic-tree for PSHA by Kulkarni et al. (1984), there were branches only for elements of the seismic source model. In a subsequent study, Coppersmith and Youngs (1986) included a node for GMMs in a logic-tree, with two branches carrying equally-weighted models (Figure 1), selected and weighted on the basis of their assumed applicability to the target region.

During the ensuing years, practice evolved to generally include more branches in groundmotion logic-trees, facilitated in part by the availability of ever-increasing numbers of published GMMs (e.g., Douglas and Edwards, 2016) and prompted by the realization that a ground-motion logic tree with only two or three branches was unlikely to capture the range of epistemic uncertainty in ground motions. A consequence of populating logic-tree branches with many GMMs was that it often became necessary to apply adjustments for incompatible definitions of explanatory variables, which made PSHA calculations more cumbersome and also led to inflated measures of aleatory variability due to propagation of the variability inherent to these empirical adjustments (e.g., Bommer et al., 2005).

Important parallel developments were related to refined understanding of the nature of uncertainty in GMMs. A GMM predicts a distribution of values of a specific ground-motion parameter, such as spectral acceleration at a particular oscillator period, as a function of explanatory variables that characterize the earthquake source (magnitude, depth, styleof-faulting), the travel path (distance) and the site ( $\mathrm{V}_{\mathrm{s} 30}$, depth of sediments). The distribution of ground-motion amplitudes is defined by the median values and the logarithmic standard deviation, generally referred to as sigma, which is interpreted as 
representing inherent randomness or aleatory variability in the ground motions. However, it is recognized that sigma includes elements of epistemic uncertainty, which could be reduced through improved characterization of some feature of the source, path or site. Consequently, sigma is always defined relative to a particular model and for this reason may often be referred to as apparent randomness, or apparent aleatory variability (e.g., Strasser et al., 2009; Stafford, 2015).

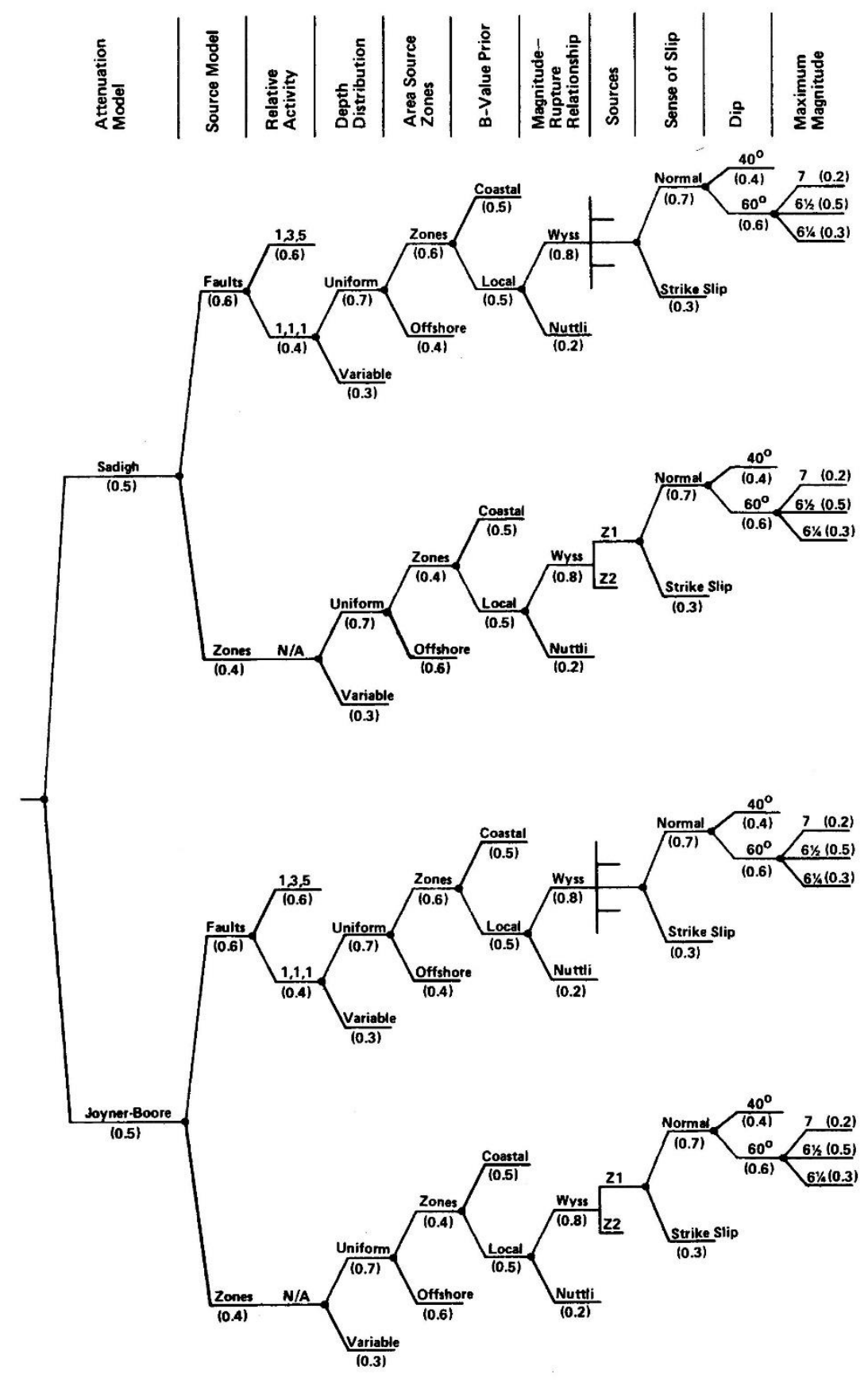

Figure 1. Logic-tree for PSHA with a single two-branch node for GMMs, referred to as 'Attenuation Model' (Coppersmith and Youngs, 1986). 
Ground-motion logic-trees evolved to include nodes for the epistemic uncertainty in both the median predictions and in sigma. One way that this was done was for the PSHA conducted for the proposed Yucca Mountain waste repository in Nevada (Stepp et al., 2001), which represented a departure from discrete branches (which approximate a continuous distribution). In that project, the ground-motion experts were required to define distributions of median values and sigma, referred to as $\sigma_{\mu}$ and $\sigma_{\sigma}$.

Another significant development in ground-motion modeling for site-specific PSHA studies was the move to including site response analysis to capture the influence of nearsurface stratigraphy rather than applying the generic amplification factors embedded in GMMs and conditioned on proxy parameters such as $V_{s 30}$ and depth of sediments. Common practice is now to define a reference rock horizon to which the ground-motion logic-tree is calibrated and then to combine the reference rock hazard with the sitespecific amplification factors for the overlying material (e.g., Rodriguez-Marek et al., 2014). Within this framework, the site-to-site component of the apparent variability in the GMM is represented as epistemic uncertainty in the site response characteristics, which necessitates the use of partially non-ergodic or single-station sigma for the reference rock GMM. For such circumstances, the ability to separate median model and sigma in the logic-tree is a very clear advantage since GMMs with fully ergodic sigma (Anderson and Brune, 1999) may be adopted for median predictions and combined with single-station sigma.

For about two decades, the common practice was to populate logic-tree branches with published GMMs and to assign relative weights to reflect their applicability to the target region as well as the general confidence in the models performing well in terms of providing robust predictions for the rupture scenarios relevant to the hazard calculations. The objective of a ground-motion logic-tree, however, is not to organize and rank GMMs but rather to predict a distribution representing the CBR of ground-motion amplitudes for given combinations of magnitude, distance and other independent variables. The relationship between weights on models occupying logic-tree branches and the resulting distribution of ground-motion amplitudes is generally not clear and requires examination through trellis plots (e.g., Stewart et al., 2015), composite model construction (Scherbaum et al., 2005) or advanced visualization tools such as Sammon's maps (Scherbaum et al., 2010). Such visualizations can reveal that the resulting distributions of ground-motion amplitudes are often much narrower than might be expected, at least for some combinations of magnitude and distance: including many branches on the logic-tree populated with several published GMMs does not automatically lead to the effective representation of epistemic uncertainty in ground motions. At the same time, it has become accepted that even in a data-rich region, the range of predictions obtained from a suite of models derived from the common database is unlikely to capture the full range of possibilities. This has prompted formulations for additional epistemic uncertainty to be added in the form of additional logic-tree branches when the models are applied in the 
region for which they were derived (e.g., Al Atik and Youngs, 2014). Clearly, if a suite of GMMs derived specifically for a region are not considered to capture the full range of epistemic uncertainty when applied locally, it follows that when applied in another region the distribution must be broader. All of these issues are addressed by using approaches that have been collectively assigned the name of backbone GMM. The common feature of all approaches to which this description is applied is that the models on branches of the ground-motion logic-tree are scaled or adjusted versions of a single GMM, which is referred to as the backbone. The backbone can be scaled to cover a range that is broader than that obtained from published models and hence the objective of covering the full range of epistemic uncertainty can be achieved in a straightforward manner. Moreover, the relationship between the weights on the branches and the resulting distribution of ground-motion amplitudes is much more transparent (Atkinson et al., 2014). The backbone GMM approach therefore allows the construction of ground-motion logic-trees for which the predictions obtained from models in individual branches neither cross nor coalesce and the predictions can cover the full range of epistemic uncertainty. As a result, the logic-tree branches then satisfy the criteria of being mutually exclusive and collectively exhaustive, which are requirements for the branch weights to be treated as probabilities in the calculation of the mean hazard curve and the associated fractiles (Bommer, 2012).

From the perspective of representing the CBR of possible ground-motion amplitudes from potential future earthquakes, we believe that it is quite difficult to justify the traditional approach of assembling a ground-motion logic-tree by selecting and weighting a number of published GMMs. Nonetheless, the backbone GMM approach has met with some resistance, for which at least three motives can be identified. The first is that this is a relatively new innovation that has neither been widely accepted nor undergone regulatory scrutiny. In fact, all that is really new is the label of backbone GMM approach and there are many examples of how variations of the approach have been used for many years (e.g., Atkinson et al., 2014). For example, the approach used in the Yucca Mountain project described above, could be considered to fall within the classification of a backbone GMM method but represented as a continuous distribution rather than discrete branches. The second objection is based on the misconception that the backbone approach always means applying constant scale factors to GMMs, whereas there are numerous examples of implementations where the adjustments to the backbone model to create alternative branches have varied with magnitude and/or distance. An example is shown in Figure 2, which is from the study of Toro et al. (1997) —and therefore a rebuttal of both of these objections. The third objection is that the alternative models obtained by scaling or adjusting the backbone GMM do not possess the pedigree and rigor of published GMMs. This position binds the hazard analyst to attempt to represent a potentially broad range of uncertainty with the range of predictions obtained from existing GMMs and hinders the possibility of crafting models that are more closely calibrated to local conditions, as described in the section Ground-Motion Logic-Trees for Site-Specific PSHA below. 


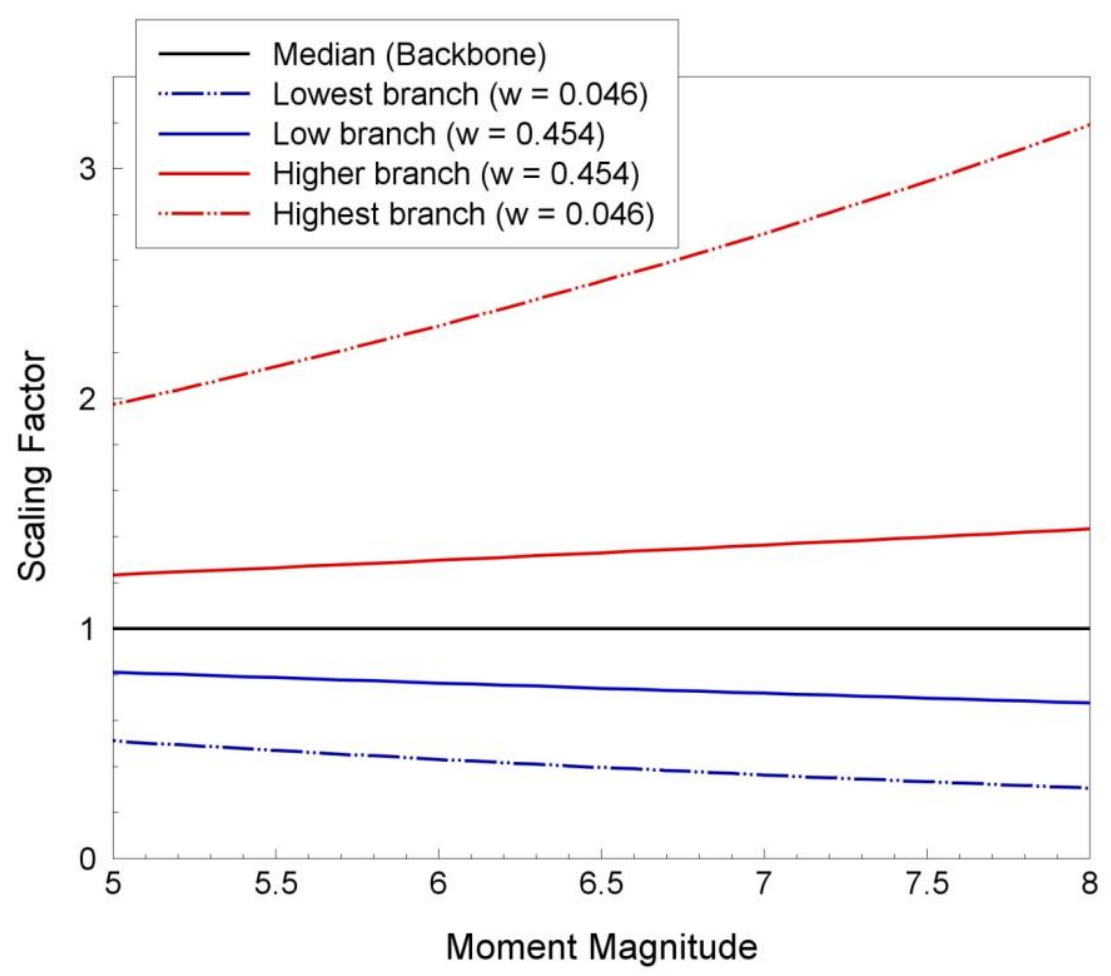

Figure 2. Magnitude-dependence scaling factors and associated branch weights for the logictree proposed for CEUS by Toro et al. (1997).

The evolution of ground-motion logic-trees for PSHA as described in the preceding paragraphs could be characterized as having focused in large part on the classification of uncertainties in ground-motion prediction and the inclusion of these uncertainties in PSHA calculations. One could argue, therefore, that the evolution from logic-trees such as one illustrated in Figure 1 to those constructed using backbone GMMs with separate nodes for medians and sigmas has been about more effectively capturing the body and range of the distribution of future ground motions. In a parallel and overlapping sequence of steps, there has also been an evolutionary process related to better capture of the center through the selection-or generation-of models applicable to the geological and geophysical characteristics of the target region and the target site. The evolution of procedures to identify GMMs that satisfy applicability criteria is described in the next section.

\section{Selecting Applicable GMMs}

Although appreciable emphasis is often placed on ensuring the capture of epistemic uncertainty in PSHA, the first and most fundamental objective is to construct a groundmotion characterization model that is well-suited to the target region and site (and then to characterize the associated uncertainty with regards to that basic goal). In this section, 
we provide a brief overview of how the issue of applicability of GMMs to the target region and site has been addressed in practice.

\section{Selection criteria for quality and stability}

Working within the framework of populating the branches of the ground-motion logic-tree with multiple GMMs but adjusted to target-region conditions, Cotton et al. (2006) proposed a set of criteria for selecting GMMs to be used in PSHA. Their premise was that to avoid bias due to factors such as familiarity or availability the selection should begin by assembling all available GMMs and then applying objective criteria to exclude those not considered fit for purpose. The suite of GMMs surviving the application of these criteria would not automatically define the models that would be used to construct the logic-tree, but rather a reduced pool from which to make the final selection. Bommer et al. (2010) expanded the list of exclusion criteria and made them more specific, but the general approach was the same. Most of the selection criteria specified in both studies were related to the inherent quality of the GMM (in terms of peer-reviewed publication, size of the database used to derive the model, appropriate definitions for explanatory variables, etc.) and the stability of the model when extrapolated beyond the magnitude-distance space covered by the database (nonlinear scaling with magnitude, magnitude-dependent geometrical spreading, etc.). In both studies, only a single criterion was specified related to regional applicability and was actually aimed only at excluding the most obviously inapplicable models: for example, not considering subduction-zone GMMs for modeling ground-motions from shallow crustal earthquakes.

The Cotton et al. (2006) study was related to seismic hazard analysis in central Europe. After selecting a suite of GMMs deemed to be reliable for general application, these were subjected to a series of adjustments for parameter compatibility and then full host-totarget region adjustments following the hybrid-empirical approach of Campbell (2003). Within that framework, it was not necessary to focus particularly on selecting GMMs that would be applicable to the target region beyond the exclusion of patently inappropriate models as described above.

\section{Testing GMM applicability using local data}

In practice, GMMs have often been adopted for seismic hazard studies on the basis of vague notions of applicability related to assumed regional analogies and similarity of tectonic environments. Such notions can often be misleading since the basis for defining regional analogs may not be related to ground-motion characteristics. The clearest example of which perhaps is the concept of Stable Continental Regions (SCRs), defined expressly and exclusively for the purpose of estimating maximum magnitudes for PSHA (Johnston, 1996). Ground-motion attenuation characteristics, as inferred from intensities, 
have been shown to vary significantly from one SCR to another (e.g., Bakun and McGarr, 2002).

A more quantitative and objective basis for identifying GMMs applicable to a particular region is to explore how well a particular model performs against ground-motion recordings from that region (e.g., Scherbaum et al., 2004; Stafford et al., 2008; Scherbaum et al., 2009; Kale and Akkar, 2013; Cremen et al., 2020). Proposals have also been made to use intensity data for such testing in combination with empirical conversions between intensity and ground-motion parameters (Delavaud et al., 2009).

In many cases, the local ground-motion recordings will be limited in number and moreover predominantly from small-magnitude events. Beauval et al. (2012) have demonstrated that testing with small-magnitude recordings will not necessarily be indicative of the relative performance of GMMs at larger magnitude (which will generally govern the results of PSHA). While intensity data may be available from larger earthquakes, the uncertainties associated with selecting an appropriate model to translate instrumental ground-motion parameters into intensities, and the variability associated with such models, limit the confidence that can be placed upon inferences made using such data.

\section{Adjustable ground-motion models}

An alternative to selecting existing GMMs on the basis of being locally applicable is to develop models that are intended to be easily adjustable to source and path conditions in a given target region. In recent years, a few studies have produced models that are specifically intended to be adjustable. These studies directly address the link between the Fourier and response spectral domains by making the derived models operate on parameters typically used to describe the Fourier amplitude spectrum (FAS), in particular, $\Delta \sigma$ and $\mathrm{k}$. For example, Bora et al. $(2014 ; 2015)$ worked with empirical ground-motion data directly to derive FAS models that when combined with a separate duration model allow for the prediction of response spectral ordinates. The resulting models can take input values of stress drop $(\Delta \sigma)$ or site kappa $\left(\mathrm{K}_{0}\right)$ when making response spectral predictions that would then be calibrated to target-region source and site characteristics. While this is a potentially very useful approach, at this moment in time it is considered these publications reflect a proof of concept rather than models that would be used in practical applications.

A similar objective, implemented in a simpler manner, was addressed in the work of Yenier and Atkinson (2015) and more recently by Hassani and Atkinson (2018). These studies make use of the theoretical model for the FAS to generate simulated response spectral ordinates for a very large number of combinations of stress drop and kappa. Parametric equations are then fit to various source, path and site terms in order to obtain 
a response spectral model that takes stress drop and kappa as input parameters. These latter models are also explicitly designed with a 'plug and play' framework such that alternative models for site response and path scaling can be included along with a local calibration parameter to tune the predictions to a particular region (note that this calibration parameter is effectively just a bias correction). Figure 3 shows that this approach can provide predictions that are generally in good agreement with GMMs derived in a more traditional manner. In particular, the agreement is very strong for the most 'point-source like' scenarios (i.e., smaller magnitudes) for which the comparison is made. The Hassani and Atkinson (2018) model uses an equivalent point-source approach for modelling motions from the largest events and so the deviations at the larger magnitudes are not particularly surprising. However, the potential benefits of this approach can be seen when modifying these predictions for alternative combinations of stress drop or kappa, as shown in Figure 4.

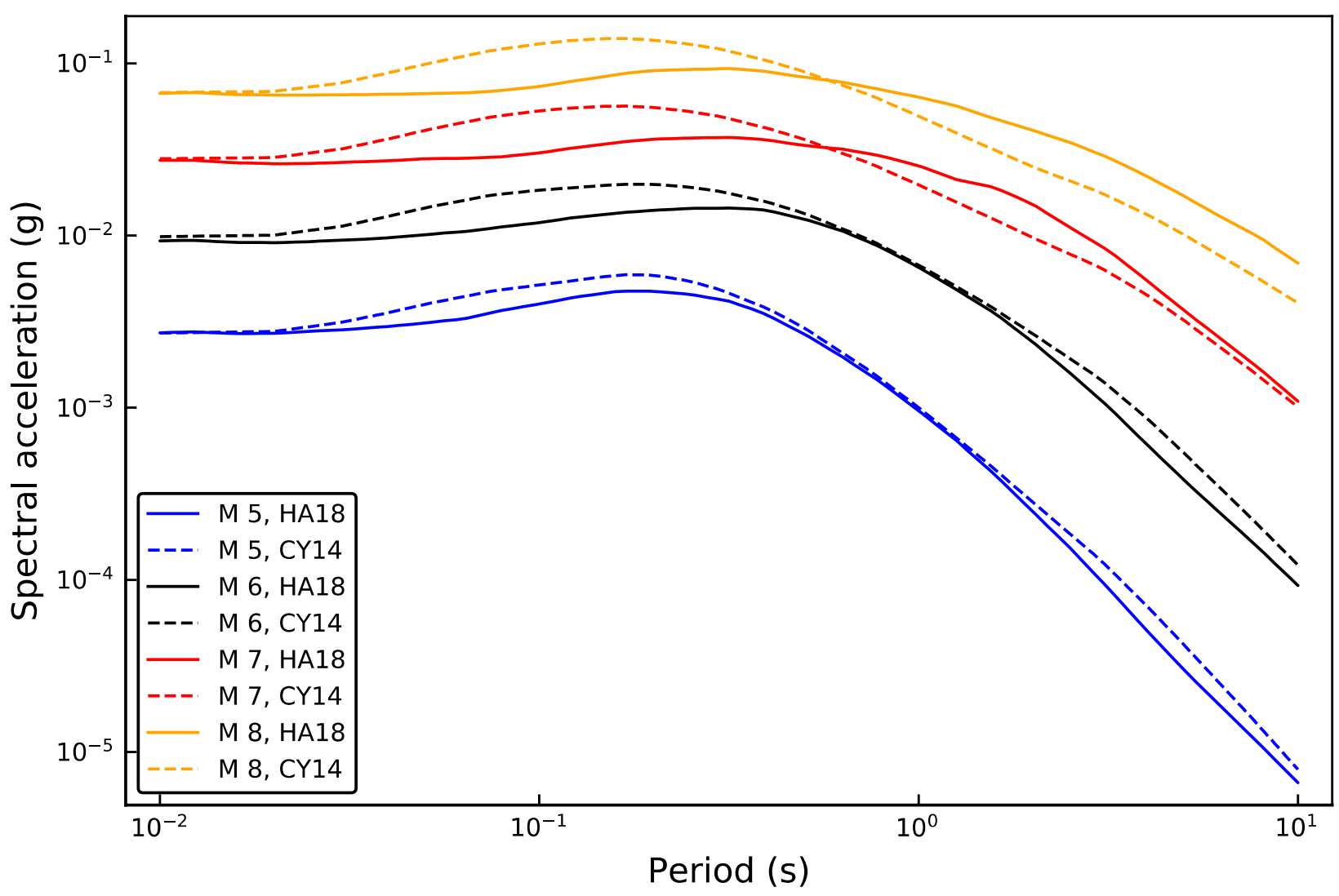

Figure 3. Comparison of response spectral predictions using the Chiou and Youngs (2014) and Hassani and Atkinson (2018) ground-motion models (the latter using just the linear site amplifications of Boore, 2016). All spectra are for a rupture distance of $100 \mathrm{~km}$ and for a rock site with $\mathrm{V}_{\mathrm{S} 30}=760 \mathrm{~m} / \mathrm{s}$. For the Hassani and Atkinson (2018) model, the stress drop is set at 70 bars and kappa at $0.04 \mathrm{~s}$, these being the authors' recommended values for California. 

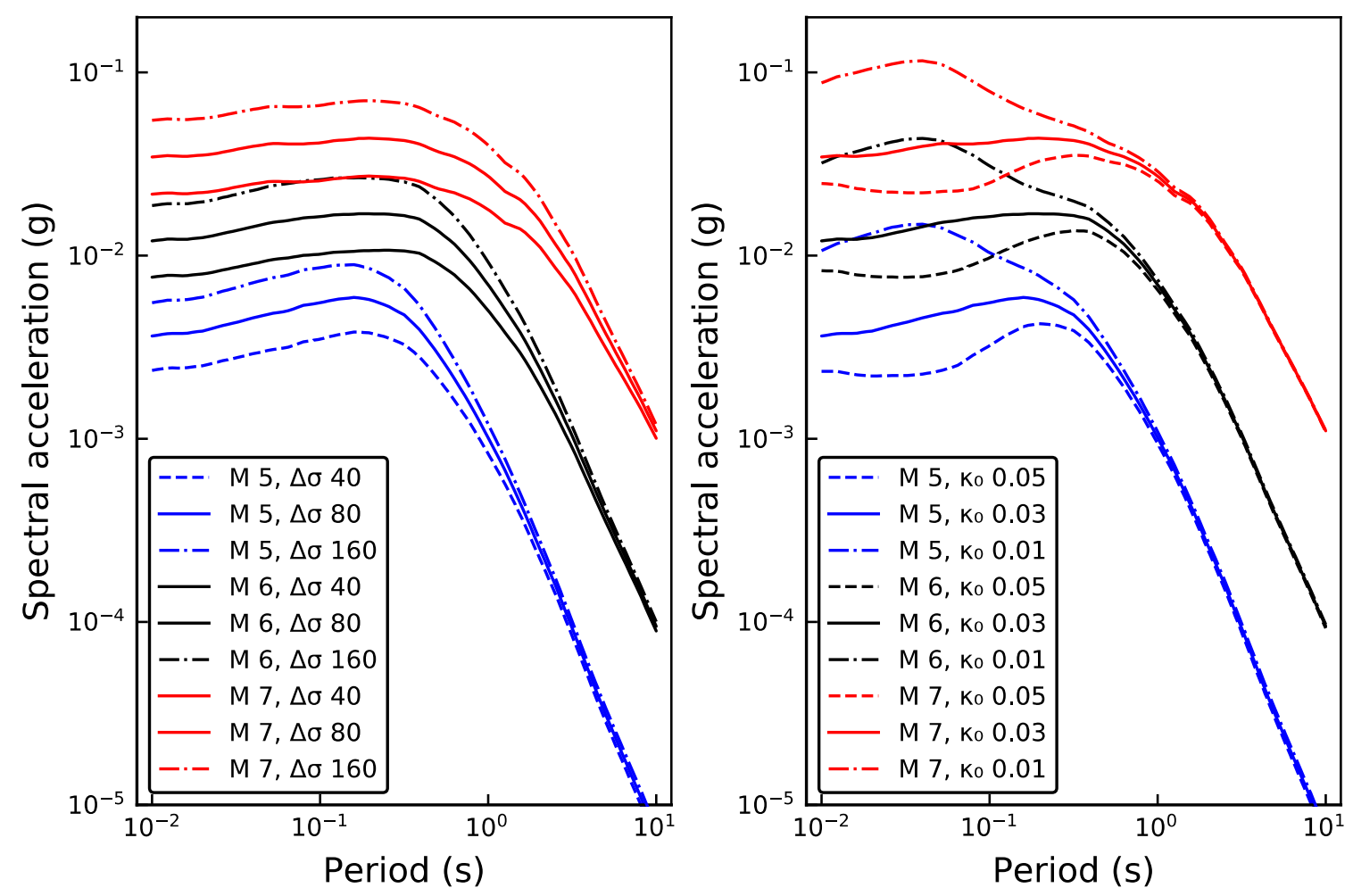

Figure 4. Variation in response spectral predictions for a number of stress drop (left) and kappa (right) values using the model of Hassani and Atkinson (2018), with tuning for application in California following the guidance in their article.

The study of Hassani and Atkinson (2018) offers some significant advantages since their modelling approach effectively involves running many SMSIM (Boore, 2003) simulations for a broad range of FAS parameters. This same work is typically required when making a parametric adjustment (see below) and so the relative impact of changes to particular parameters can be readily investigated using their model. This feature of their approach is useful in the context of establishing scaling factors for backbone models. However, the use of a point-source representation for earthquake sources is clearly a significant approximation and therefore not ideal for a site-specific hazard study. In particular, nearsource rupture effects, such as the amplification of motions on the hanging-wall of a dipslip rupture (e.g., Donahue and Abrahamson, 2014), can never be captured effectively with such models.

\section{Ground-Motion Logic-Trees for Site-Specific PSHA}

Based on the considerations discussed in the previous sections, we propose the following as the optimal procedure for developing a GMC logic-tree for site-specific PSHA: 
1. Adopt the backbone GMM approach. We have presented several arguments in favor of this approach but in reality it is already being widely adopted in site-specific PSHAs and we believe that it would actually be more difficult to justify using the traditional approach of populating the branches with multiple GMMs. Effective capture of the CBR of TDI regarding ground-motion amplitudes from future earthquake scenarios cannot be assured when using multiple published GMMs, whereas the backbone approach greatly facilitates meeting this fundamental objective.

2. Select a suite of potential backbone GMMs that can be extrapolated in a stable manner. As has been proposed formally by Cotton et al. (2006) and Bommer et al. (2010), the starting point should be to identify all available GMMs that are appropriate to the seismic source type (i.e., crustal or subduction), and then apply pre-established exclusion criteria. As discussed previously, these criteria will mainly focus on removing models that are poorly constrained or have functional forms that are unlikely to produce reliable predictions when extrapolated to the limiting magnitude-distance combinations considered in the hazard integrations. In order to ensure that the models include near-source effects, it is expected that the suite of models will be comprised of empirical GMMs or potentially physics-based GMMs that explicitly model these effects (when such models become sufficiently robust).

3. Apply host-to-target region adjustments to the final backbone GMM, including ranges of epistemic uncertainty for each adjustment. Once a final backbone GMM is selected, a series of adjustments should be made for differences between the source and path characteristics in the host and target regions. Adjustments can also be made from the reference rock profile implicit in the backbone GMM to the target site profile. A node should be added to the logic-tree for each of the adjustments, with branches capturing the range of uncertainty associated with both the host- and target-region estimates of the value or model element in question (although commonly the uncertainty will be assigned to the target-region, both because that is where it will be larger and also to avoid excessive numbers of branch combinations). In this way, the full range of epistemic uncertainty can be built up in a systematic and tractable manner. If several nodes, each with many branches, are needed then it is a straightforward matter to re-sample the branches from several or all nodes into a simpler representation (e.g., Miller and Rice, 1983) even though this means that the contributions of individual nodes can no longer be identified through disaggregation.

The attentive reader will notice that one detail is omitted from this brief list: from the suite of candidate GMMs identified in step \#2, only one is used in step \#3. In practice, the emphasis will have generally been placed on selecting the model best suited to the region in question, or in other words, the GMM considered most applicable. In this new paradigm, the applicability of the selected GMM—beyond avoiding clearly inappropriate models 
such as an in-slab subduction model for use in a shallow crustal seismic source-is no longer a consideration. The reason is very simply that the adjustments made in step \#3 are designed precisely to render the backbone GMM applicable to both the region and the site-and to capture the uncertainty in the source, path, and site characteristics in the target region (and also accounting for the uncertainty in these characteristics in the host region). This is a perhaps a subtle but nonetheless very important difference, because the criterion for selecting the final backbone GMM is not its applicability to the target region but rather how amenable the model is to being adjusted so as to match the targetregion conditions. We return to this point a little later.

Another issue regarding which there is a lack of detail in the brief list above is how to actually make the adjustments to render the backbone GMM more applicable to the target region. A detailed description of each of the possible adjustments for source, path and site differences is beyond the scope of this paper, but an overview of the general approach is needed. There are essentially two options, these being an empirical approach and a parametric approach. The former involves adjusting terms in the GMM in order to better match recordings from the target region, informed initially by residual analysis. This approach has been given the name of referenced empirical adjustments (Atkinson, 2008). An attraction of this approach is that it is fairly simple to implement but it also suffers from important limitations. For site-specific PSHA studies, in which it should be considered indispensable to capture local amplification effects, this is only possible if there exists a database of ground-motion recordings from the site itself, which is unlikely to be the case. If the available recordings are predominantly from smaller magnitude earthquakes, the referenced empirical approach is also not well equipped to constrain source characteristics in the target region.

Our preference is to apply parametric adjustments using the hybrid empirical method (HEM) of Campbell (2003), as illustrated schematically in Figure 5. The essence of the HEM is to define the source, path and site parameters that predict FAS in the host and target regions, the parameters being stress drop $(\Delta \sigma)$, focal depth, geometric spreading, path quality factor $(\mathrm{Q})$, site shear-wave velocity $\left(\mathrm{V}_{\mathrm{S}}\right)$ profile, and site kappa $(\mathrm{K})$. The parameter values may be retrieved from published studies, from inversions of groundmotion recordings or from inversions of predicted response spectral ordinates (in the host region). In the original proposal of Campbell (2003), adjustments would be made for hostto-target differences in all of these characteristics simultaneously. However, it is now common practice to adjust for some of the source, path, and site differences, and also to make these adjustments individually. This allows the uncertainty in each of the adjustments to be captured through the use of logic-trees, as mentioned earlier. In this way, the total epistemic uncertainty in the final model applied to the target region is built up in a transparent and tractable manner. 


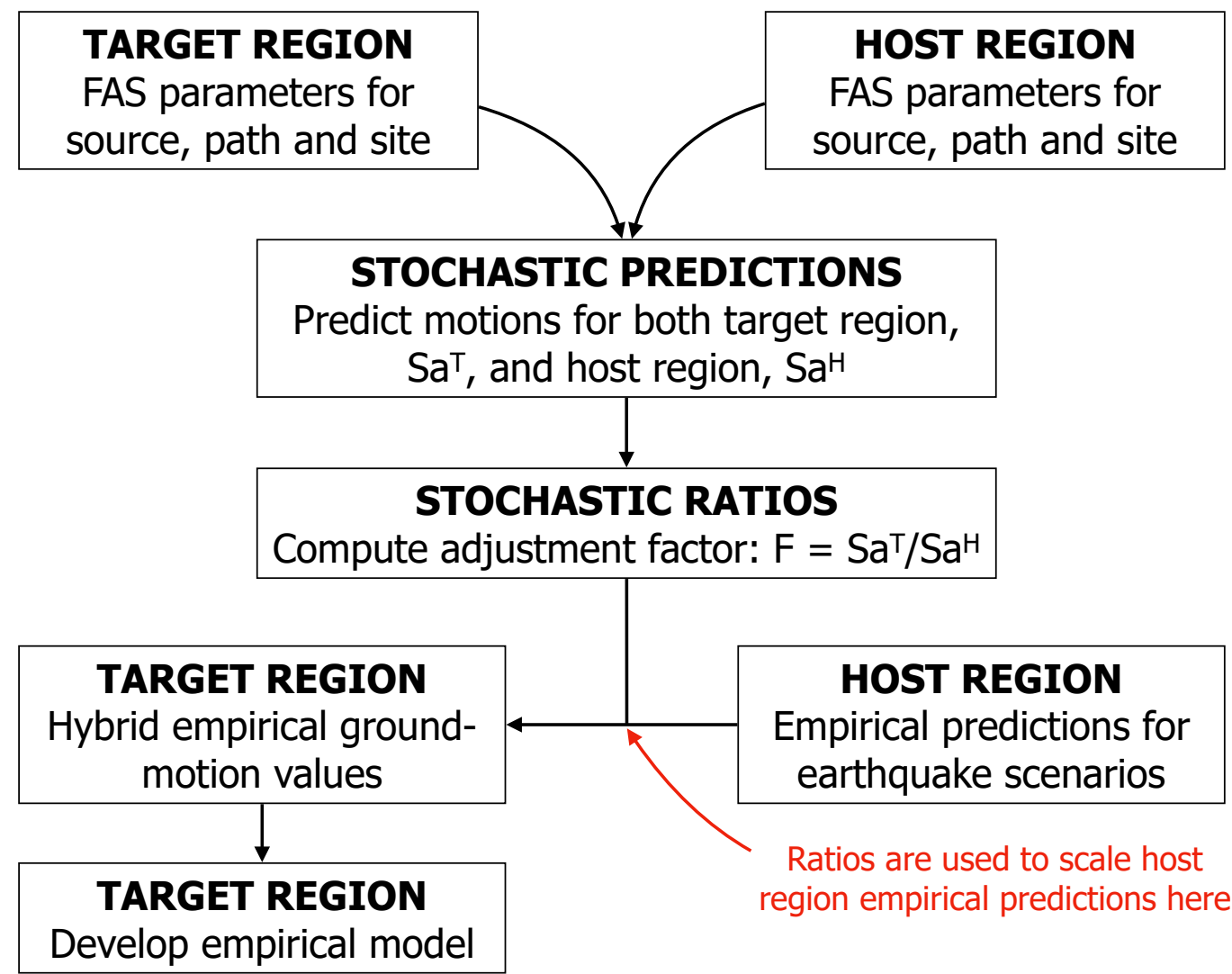

Figure 5. Schematic illustration of the hybrid empirical method for adjusting an empirical GMM to match conditions in the target region and at the target site.

In building a GMC logic-tree in this way, it becomes clear that the important feature of the backbone GMM is not that it is applicable to the target region, but rather than it is well suited to being adapted or adjusted to the target region and site through parametric adjustments using the HEM.

\section{The Characteristics of Adaptable Ground-Motion Models}

Within the proposed framework for the construction of GMC logic-trees for site-specific PSHA, the concept of how applicable a GMM may be to the target region is no longer a consideration in selecting the backbone model. Rather, a model is sought that has the potential to be adapted to match the source and path characteristics of the target region, as well as to reflect the dynamic response characteristics of the target site. The characteristics of an adaptable GMM can be grouped into three categories: 
- Host-region parameterization. For each source, path, and site parameter that it is to be adjusted, it is essential that well constrained values of these parameters in the host-region can be determined. This is important in order to have confidence in the starting point for each host-to-target region adjustment and to avoid introducing bias resulting from errors in the host-region parameterization. For example, in terms of host-region site characteristics, the GMM should explicitly model the influence of $V_{S 30}$ rather than being based on site classes defined by ranges of $V_{S 30}$. However, $V_{S 30}$ alone is insufficient: it should be possible to define a generic profile, based on measured $V_{s}$ profiles, that extends considerably deeper than the top 30 meters. In this way, the site effects implicitly included within the GMM are associated with a well-defined near-surface and crustal profile. The estimation of the parameters that characterize the host region is not a trivial task and will generally involve appreciable uncertainty. It is also important that the hostregion characteristics are estimated collectively rather than selecting individual parameters from separate studies because of the strong correlations and tradeoffs among the various source, path and site parameters.

- Isolated influence of individual factors. The influence of each source, path and site parameter on the predicted response spectral ordinates should be clearly identifiable. This is to allow for adjustments to be made for each factor individually, making the overall adjustment and the incorporation of epistemic uncertainty clear and tractable. By way of illustration, consider the dependence of spectral accelerations on distance, which should include terms for both geometric spreading and anelastic attenuation rather than some single equivalent term that attempts to reflect the combined attenuation. Moreover, ideally the distancedependent and distance-independent contributions to anelastic attenuation should be decoupled.

- Functional form that reflects theoretical scaling. Since the adjustments are made using the HEM, the scaling of ground-motion parameters with respect to each factor to be adjusted should be consistent with seismological theory as embedded in stochastic simulations (e.g., Boore, 2003). As has been demonstrated in detail by Bora et al. (2016), the relationship between the ordinates of FAS and response spectral acceleration $[\mathrm{Sa}(\mathrm{T})]$ is complex. Response spectral ordinates, as well as PGA (peak ground acceleration), reflect a range of FAS, particularly for higher oscillator frequencies. Consequently, the hybrid empirical approach can be expected to work better when the GMM predicts scaling of ground-motion parameters with source, path and site characteristics in a manner similar to the scaling implicit in stochastic simulations.

The last point is particularly important and is fundamental to the concept of adaptability. This criterion relates to the extent to which the functional terms of a model are relatable to physical attributes of the ground-motions, as described by the simplified Fourier spectral representation of far-field shear-waves. This includes having a model whose 
functional terms scale in a manner that is consistent with theoretical expectations. Note that this places a relatively large emphasis upon the robustness and reliability of the standard model for Fourier spectral ordinates-and particularly on the relevance of simplified representations of the source (both as a point-source, or equivalent pointsource, and as a simple single or double-corner source spectrum); it is important that the same type of FAS works in both the host and target regions. However, as discussed previously, given that this framework is used to identify the properties of the target region under the parametric framework there are significant advantages from an adaptability perspective of having a host GMM that is largely consistent with the theoretically implied scaling.

In order to provide a concrete example of this point, consider the magnitude scaling of the NGA-West models (the main points here relate to the parameterization of both the NGA-West1 and NGA-West2 models; Abrahamson et al., 2008, Gregor et al. 2014). Following the work of Fukushima (1996), and using the basic concept of an earthquake source spectrum with at least one corner frequency that scales with magnitude - such as the omega-squared spectrum (Aki, 1967), it can be shown that the magnitude scaling of response spectral ordinates should behave as in Figure 6.

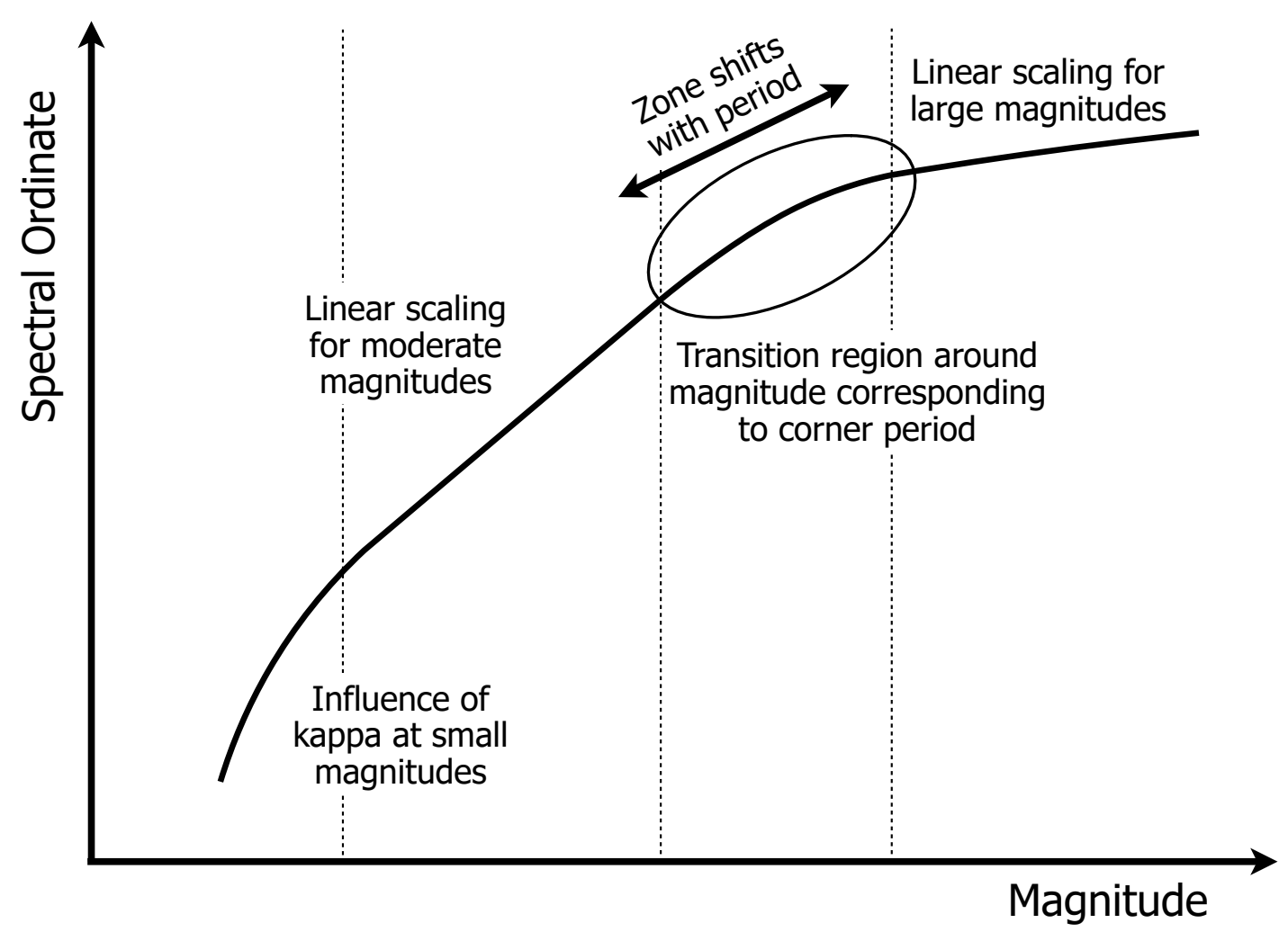

Figure 6. Schematic illustration of the key features of magnitude scaling that arise from consideration of point-source FAS. The transition zone moves to larger magnitudes as the response period increases. 
Similar insights to ground-motion scaling with magnitude can be found in Douglas and Jousset (2011) and Baltay and Hanks (2014). Each of the NGA-West models adopt a different functional form that is sufficiently flexible to capture most of the key features of the theoretical scaling shown in Figure 6. However, not all of the models are able to replicate all expected features of the scaling shown, and those that can often do so through the use of a relatively large number of degrees of freedom (e.g., multiple piecewise segments with coupled hinge magnitudes and slopes). The principal exception to this is the model of Chiou and Youngs $(2008 ; 2014)$ for which the functional form is motivated by theoretical considerations pertaining to the scaling of earthquake source spectra in the Fourier domain (Chiou and Youngs, 2008). For this model, each parameter in the base magnitude scaling has a clear physical meaning that captures all of the features shown in Figure 6 (Chiou et al, 2010), apart from the kappa-influenced deviations at small magnitudes (outside of the range of interest for the initial NGA-West project).

The identification of an adaptable GMM is illustrated in the following section for the case of models applicable to active crustal regions.

\section{Adaptable Ground-Motion Models for Active Crustal Regions}

When looking to identify adaptable models that are well-suited to the role of a backbone model, we must first identify potential candidates accounting for the features highlighted previously. Despite the very large number of empirical GMMs that have been published (Douglas, 2019), only a very small portion of these models satisfy the basic requirements for use within a high-level hazard analysis. In particular, it remains uncommon for models to explicitly include functional terms to adequately capture near-field finite-rupture effects, and many models are also unable to appropriately account for the effects of nonlinear site response. These features of GMMs arise for rupture scenarios that often make important contributions to seismic hazard, but that are poorly constrained within empirical databases. It is therefore important to adopt 'next generation' approaches to model development that also include numerical or theoretical constraints to ensure robust performance of the model under extrapolation to scenarios not well-represented by the empirical data.

Of the models that do satisfy the criteria for consideration as backbone models, those arising from the NGA-West2 project (Bozorgnia et al., 2014) are the strongest candidates. Of the alternative candidates, such as the models of Akkar, Sandıkkaya and Bommer (2014), Bindi et al. (2014) and Kotha et al. (2016) effectively arising from the RESORCE project (Akkar, Sandıkkaya, Şenyurt et al., 2014), the global model of Cauzzi et al. (2015), or the recent models for Iran (Sedaghati and Pezeshk, 2017; Farajpour et al., 2019; Darzi et al., 2019), each of these can be shown to have problems associated with their adaptability. Specifically, the ergodic regional (Akkar, Sandıkkaya and Bommer, 2014; 
Bindi et al., 2014) or global (Cauzzi et al., 2015) models suffer from not being able to define an underlying crustal profile representative of the regions they cover, and also do not consider other known regional differences that compromise the ability to define unambiguous host parameters (Stafford, 2014). Many of these models also have simplified treatments of site effects (e.g., Cauzzi et al., 2015; Kotha et al., 2016; Darzi et al., 2019) using linear scaling with $V_{S 30}$ or discrete site classes. Furthermore, none of these alternative candidates account appropriately for finite-rupture effects.

Considering the NGA-West2 models, a common feature for all of these candidates is their shared ergodic database. At face value the use of such an ergodic database suggests problems from an adaptability perspective as it is hard to argue for the existence of a generic underlying crustal profile that is required to define host impedance effects or anelastic attenuation (both path and site components). However, the models of Abrahamson et al. (2014), Boore et al. (2014), Campbell and Bozorgnia (2014) and Chiou and Youngs (2014) all include regionalization terms that can be used to target the predictions of these models to certain regions. It is therefore possible to target the predictions of these models to represent scaling associated with a generic crustal profile such as that of Boore (2016) appropriate for the western US.

From the four NGA-West2 models that account for regionalization effects (and nonlinear site effects), it can be argued that the Chiou and Youngs (2014;) model is best suited for use as a backbone model for at least two strong reasons. The first point relates to the parameterization of the magnitude scaling previously noted. Because of the theoretical basis of their functional form, it is possible to associate the coefficient $c_{M}$ of their model with stress drop and to therefore make corrections for stress drop differences by directly targeting a single parameter of their model. Figure 7 demonstrates this ability by comparing the change in response spectral shape corresponding to a factor of two change in stress drop. For reference, the adjustable GMM of Hassani and Atkinson (2018) is shown along with the adjusted predictions of the Chiou and Youngs (2014; CY14) model. Recall that the Hassani and Atkinson (2018) model is effectively an empirical regression on a very large number of equivalent point-source stochastic simulations the same as those performed within the HEM. Specifically, for magnitudes below $c_{M}$ CY14 has steep scaling associated with being below the source corner-frequency of the FAS. For larger magnitudes we are above the corner frequency and the scaling is much flatter. The parameter $c_{M}$ is therefore the magnitude associated with a given corner frequency. As the corner frequency scales with the ratio of stress drop and seismic moment (Boore, 2003), we can define changes in magnitude (and hence changes to $c_{M}$ ) that have the same impact as changes in stress drop.

Figure 7 demonstrates that if one wished to make adjustments to the Chiou and Youngs (2014) model to account for host-to-target differences in stress drop then one can 
capitalize upon the theoretical basis of their functional form rather than enter into hybrid empirical adjustments.
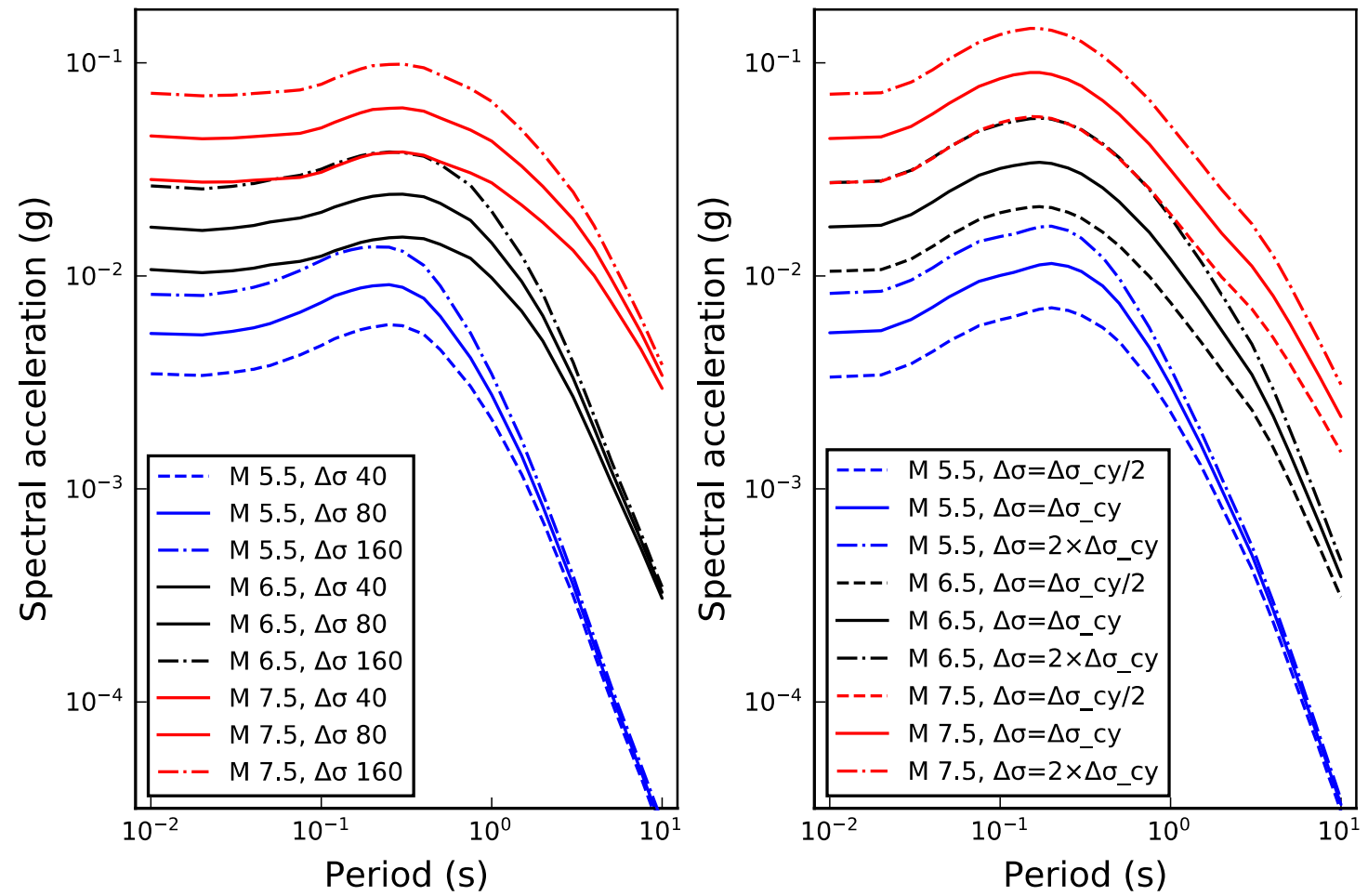

Figure 7. Comparison of the change in spectral shape arising due to a change of stress drop by a factor of 2. The left panel shows the predictions of the Hassani and Atkinson (2018) adjustable model while the right panel shows how the equivalent scaling can be obtained by targeting the $c_{M}$ parameter of the Chiou and Youngs (2014) model.

The second significant advantage of the Chiou and Youngs (2014) model over the other NGA-West2 models relates to the parameterization of anelastic attenuation effects. There are two favorable aspects to note here. To begin, the far-field geometric spreading coefficient in Chiou and Youngs (2014) is fixed to a theoretically consistent value of -0.5 associated with cylindrical spreading. This means that anelastic attenuation effects that dominate distance scaling at large distances are known to be relative to this particular geometric spreading rate, thus helping to decouple the geometric and anelastic components of the path scaling. However, the more important feature of the Chiou and Youngs (2014) parameterization is the inclusion of magnitude dependence of the anelastic attenuation. Magnitude dependence in anelastic attenuation arises for the same reason that scenario dependence arises in linear site response. The high-frequency filtering due to anelastic effects limits the bandwidth of Fourier spectral ordinates that can contribute to response spectral amplitudes (Stafford et al., 2017). The magnitude dependence of the anelastic attenuation in the Chiou and Youngs (2014) model is very similar to that which arises from stochastic simulations using Fourier spectral theory 
(Boore, 2003). The reason why this is important is that, as noted earlier, the need for parametric adjustments is often inferred on the basis of Fourier spectral inversions of small magnitude data. When models do not include these magnitude-dependent effects it becomes more challenging to understand whether observed host-to-target differences in anelastic attenuation are a physical reality, or whether they are an artifact of using observations from different magnitude ranges. To the authors' knowledge, the Chiou and Youngs (2014) model is currently the only GMM that includes this magnitude dependence within the anelastic attenuation terms.

The net result of the above considerations is that we believe that the GMM that is currently the most adaptable and suitable for use as a backbone model is that of Chiou and Youngs (2014). The key features of the model are its strong theoretical basis with respect to magnitude scaling that enables host-to-target differences in stress drop to easily be accounted for. The treatment of distance scaling is also a strong feature, particularly for far-field scenarios where geometric and anelastic contributions are cleanly decoupled and are physically consistent. The model includes regionalization terms for site effects and can be associated with a robust crustal profile appropriate for the western US. All of these features are important from an adaptability perspective and allow for host-to-target differences to be developed in a relatively straightforward manner.

By way of illustration, Figure 8 shows a GMC logic-tree for median spectral accelerations in reference rock due to crustal earthquakes that was developed in the SSHAC Level 3 PSHA for the Hanford site (PNNL, 2014). Figure 8a is the logic-tree for the $V_{S-K}$ adjustments to the reference rock, capturing uncertainty in the host-region site kappa and in the estimation of the target-region site characteristics. In Figure $8 \mathrm{~b}$, which is the logictree for median motions (constructed using the CY14 model as the backbone GMM), the 108 branch combinations from the upper logic-tree are re-sampled into a single node with seven branches. This logic-tree contrasts starkly with the single two-branched node in Figure 1, confirming how far GMC modeling has evolved over the years.

\section{Discussion and Conclusions}

The state of practice of constructing logic trees for site-specific PSHA has evolved considerably over the years, continuously coming closer to meeting the objective of capturing the CBR of TDI in terms of ground-motion amplitudes that reflects the current state-of-knowledge for the target region and site. A particularly important step in this evolution has been the increasingly widespread adoption of the so-called backbone GMM approach in which the branches of the GMC logic-tree are populated by scaled or adjusted versions of a single GMM. While applications of this approach generally involve making region- and site-specific adjustments to the backbone GMM, the focus for selecting the backbone model has generally been on the potential applicability to the 
target region. In this paper, we have put forward the case for changing the criteria for selection of the backbone GMM to its being amenable to adjustment to target-region conditions rather than any inherent applicability.

(a)
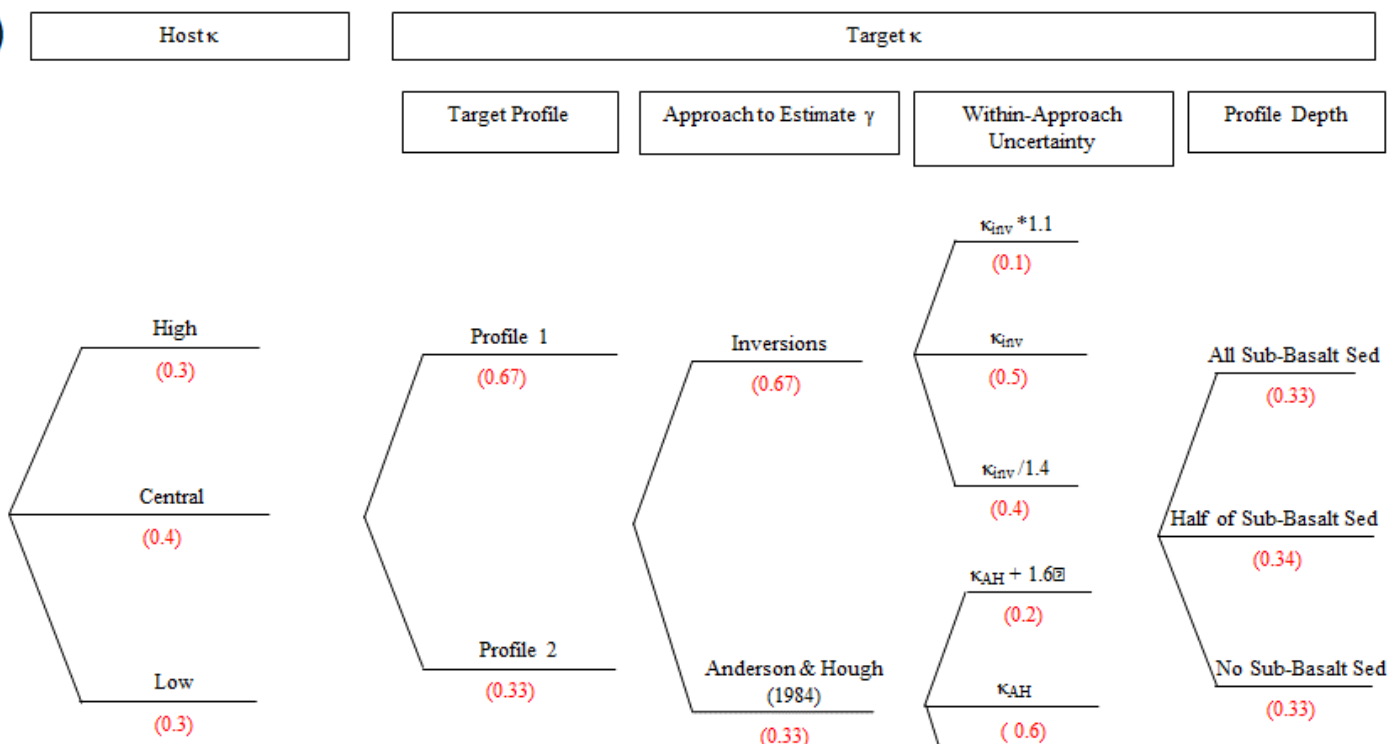

Profile Depth
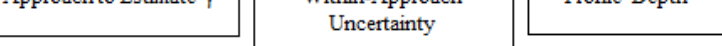

$(0.3)$
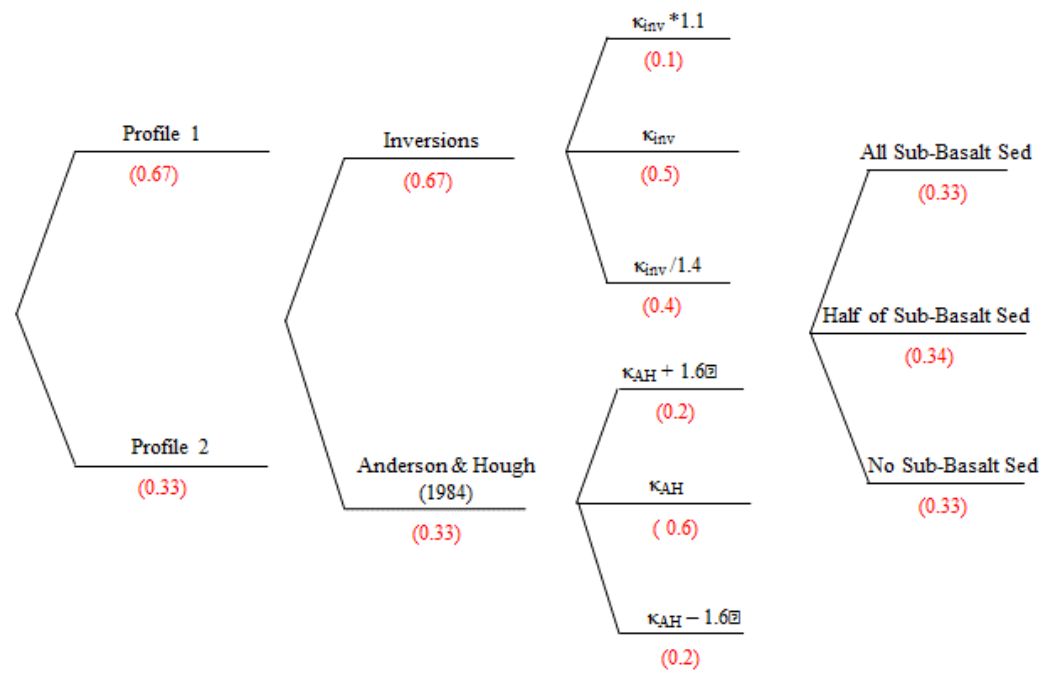

(b)

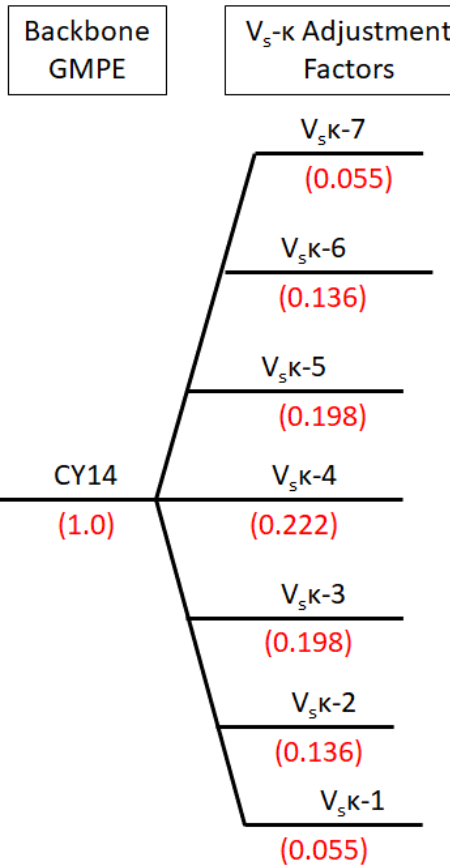

Inherent Uncertainty in Backbone Adjustments

$[\Delta \ln (\mathrm{Y}) \mid \mathrm{M}]_{9} \quad(0.0625)$

Host-to-Target Uncertainty Factors
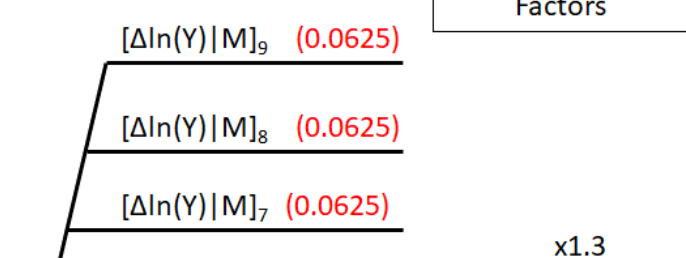
Recently, models derived from stochastic simulations have been developed precisely to enable adjustment to target-region conditions, which is entirely consistent with the general approach being proposed. However, these adjustable models lack the capacity of empirical GMMs to capture finite rupture effects in a realistic manner and are also limited in terms of how well the models can be adapted to the dynamic response characteristics of the target site. For these reasons, our alternative approach is to select an adaptable empirical GMM and then to make a series of host-to-target region adjustments for source and path differences, and then to make adjustments for local site conditions. Each adjustment can be made within a logic-tree framework with branches for alternative parameter values or models, which allows the total epistemic uncertainty in the final GMC model to be built up in a tractable manner. If this results in a large number of nodes and branches, the resulting distributions can be re-sampled with a smaller number of branches that combine the contributions from a number of nodes.

The key to implementing the proposed approach is therefore to identify a candidate GMM that is easily adapted to match target-region and target-site characteristics. An adaptable model is one for which it is possible to obtain well-constrained estimates of source, path and site parameters in the host region, with site parameters including both the shallow $V_{s}$ profile and upper crustal velocity profile. Another criterion for identifying adaptable GMMs is that the influence of individual source, path and site characteristics are clearly identifiable within the model, such that adjustments can be made for each factor individually. The third characteristic of an adaptable GMM is a functional form in which the scaling of response spectral ordinates with magnitude and distance is consistent with seismological theory as embedded in stochastic simulations (since the adjustments are made using the hybrid empirical approach). Applying these selection criteria to GMMs for active crustal regions-after an initial screening to remove models that are poorly constrained or unlikely to extrapolate to larger magnitudes in a stable manner-identifies Chiou and Youngs (2014) as the most adaptable model currently available. For sitespecific studies considering upper crustal seismicity, therefore, a possible starting point would be to simply adopt this model and focus effort and attention on the host-to-target region adjustments rather than on the selection of the backbone model. For subduction earthquakes, it would be relatively straightforward to select an appropriate backbone model given that there are far fewer models available. The subduction GMM by Abrahamson et al. (2016) could be considered an adaptable model.

The focus of this paper, as indicated by its title, has been on site-specific PSHA studies, which will generally be conducted for major infrastructure and safety-critical structures; as has been the case in many aspects of engineering seismology, much of the development reflected herein has occurred through studies for nuclear sites. However, the concept of adaptability of GMMs may have broader relevance than seismic hazard studies for nuclear power plants and waste repositories. In any site-specific PSHA, it is reasonable to expect that investment will be made in site characterization and therefore 
consideration of how easily and reliably selected GMMs may be adapted to the sitespecific profile should be a consideration, even if less effort is to be expended on calibrating the GMMs for source and path characteristics in the target region. In contrast, for seismic hazard mapping applications and regional seismic risk assessments the emphasis is more likely to be adaptability to source and path characteristics while using more generic site amplification models. In any application, the concept of adaptability can serve as a selection criterion for GMMs whether adopting the backbone approach or constructing a GMC logic-tree with multiple GMMs, although we strongly favor the former for reasons that have been expounded herein.

In terms of GMM development, our conclusion that Chiou and Youngs (2014) is the most suitable candidate as a backbone model for crustal earthquakes does not mean that all other crustal GMMs are rejected. A key element in constructing a GMC logic-tree using the backbone approach is to ensure that the range of predicted amplitudes is consistent with (or envelopes) the range of predictions from appropriate GMMs, whether this is established through trellis plots or Sammon's maps (Scherbaum et al., 2010). At the same time, we would argue for consideration to be given to adaptability criteria in the development of future GMMs. In the same way that Bommer et al. (2010) suggested that their proposed selection criteria could also be used as a checklist for GMM developers, we would suggest that future GMMs consider including features that enhance their adaptability. Alternatively, in parallel with the development of GMMs constructed for regional applicability, as has always been done, another class of empirical GMMs could be developed for the express purpose of being adapted for site-specific seismic hazard studies.

\section{Data and Resources}

No data have been used in this study. All sources of information and all references are cited in the text.

\section{Acknowledgments}

The ideas presented in this paper have been honed through extensive discussion with numerous colleagues, particularly in the context of several site-specific PSHA studies for nuclear facilities around the world. While any list will undoubtedly be incomplete in this regard, we acknowledge in particular the interactions with Norm Abrahamson, Adrian Rodriguez-Marek and Bob Youngs. We are also very grateful to Associate Editor, Ivan Wong, and to two anonymous reviewers for feedback and suggestions that helped to improve the original manuscript. 


\section{References}

Abrahamson, N. A., G. Atkinson, D. Boore, Y. Bozorgnia, K. Campbell, B. Chiou, I.M. Idriss, W. Silva, and R. Youngs (2008). Comparisons of the NGA ground-motion relations. Earthq. Spectra 24(1), 45-66.

Abrahamson, N., N. Gregor, and K. Addo (2016). BC Hydro ground motion prediction equations for subduction earthquakes. Earthq. Spectra 32(1), 23-44.

Abrahamson, N.A., W.J. Silva and R. Kamai (2014). Summary of the ASK14 ground motion relation for active crustal regions. Earthq. Spectra 30(3), 1025-1055.

Aki, K. (1967). Scaling law of seismic spectrum. J. Geophys. Res. 72(4), 1217-1231.

Akkar, S., M.A. Sandikkaya and J.J. Bommer (2014). Empirical ground-motion models for pointand extended-source crustal earthquake scenarios in Europe and the Middle East. Bull. Earthq. Eng. 12(1), 359-387.

Akkar, S., M. A. Sandıkkaya, M. Şenyurt, A. A. Sisi, B. Ö. Ay, P. Traversa, J. Douglas, F. Cotton, L. Luzi, B. Hernandez and S. Godey (2014). Reference database for seismic ground-motion in Europe (RESORCE). Bull. Earthq. Eng. 12(1), 311-339.

Al Atik, L., and R. R. Youngs (2014). Epistemic uncertainty for NGA-West2 models, Earthq. Spectra 30(3), 1301-1318.

Anderson, J. G., and J. N. Brune (1999). Probabilistic seismic hazard analysis without the ergodic assumption, Seismol. Res. Lett. 70(1), 19-28.

Atkinson, G. M. (2008). Ground-motion prediction equations for eastern North America from a referenced empirical approach: Implications for epistemic uncertainty. Bull. Seismol. Soc. Am. 98(3), 1304-1318.

Atkinson, G. M., J. J. Bommer, and N. A. Abrahamson (2014). Alternative approaches to modeling epistemic uncertainty in ground motions in probabilistic seismic-hazard analysis, Seismol. Res. Lett. 85(6), 1141-1144.

Bakun, W. H., and A. McGarr (2002). Differences in attenuation among the stable continental regions, Geophys. Res. Lett. 29(23), 36-1.

Baltay, A. S., and T.C. Hanks (2014). Understanding the magnitude dependence of PGA and PGV in NGA-West 2 data. Bull. Seismol. Soc. Am. 104(6), 2851-2865.

Beauval, C., H. Tasan, A. Laurendeau, E. Delavaud, F. Cotton, P. Guéguen, and N. Kuehn (2012). On the testing of ground-motion prediction equations against small-magnitude data. Bull. Seismol. Soc. Am. 102(5), 1994-2007. 
Bindi, D., M. Massa, L. Luzi, G. Ameri, F. Pacor, R. Puglia and P. Augliera (2014). Pan-European ground-motion prediction equations for the average horizontal component of PGA, PGV, and 5\%damped PSA at spectral periods up to 3.0s using the RESORCE dataset. Bull. Earthq. Eng. 12(1), 391-430.

Bommer, J. J. (2012). Challenges of building logic trees for probabilistic seismic hazard analysis. Earthq. Spectra 28(4), 723-1735.

Bommer, J. J., J. Douglas, F. Scherbaum, F. Cotton, H. Bungum, and D. Fäh (2010). On the selection of ground-motion prediction equations for seismic hazard analysis, Seismol. Res. Lett. 81(5), 794-801.

Bommer, J. J., F. Scherbaum, H. Bungum, F. Cotton, F. Sabetta, and N. A. Abrahamson (2005). On the use of logic trees for ground-motion prediction equations in seismic hazard assessment. Bull. Seismol. Soc. Am. 95(2), 377-389.

Boore, D. M. (2003). Simulation of ground motion using the stochastic method. Pure Appl. Geophys. 160(3-4), 635-676.

Boore, D. M. (2016). Determining generic velocity and density models for crustal amplification calculations, with an update of the Boore and Joyner (1997) generic site amplification for $\bar{V}_{S}(Z)=$ 760 m/s. Bull. Seismol. Soc. Am. 106(1), 316-320.

Boore, D.M., J.P. Stewart, E. Seyhan and G.M. Atkinson (2014). NGA-West2 equations for predicting PGA, PGV, and 5\% damped PSA for shallow crustal earthquakes. Earthq. Spectra 30(3), 1057-1085.

Bora, S. S., F. Scherbaum, N. Kuehn and P. J. Stafford (2014). Fourier spectral-and duration models for the generation of response spectra adjustable to different source-, propagation-, and site conditions. Bull. Earthq. Eng. 12(1), 467-493.

Bora, S. S., F. Scherbaum, N. Kuehn and P. J. Stafford (2016). On the relationship between Fourier and response spectra: Implications for the adjustment of empirical ground-motion prediction equations (GMPEs). Bull. Seismol. Soc. Am. 106(3), 1235-1253.

Bora, S. S., F. Scherbaum, N. Kuehn, P. J. Stafford and B. Edwards (2015). Development of a response spectral ground-motion prediction equation (GMPE) for seismic-hazard analysis from empirical Fourier spectral and duration models. Bull. Seismol. Soc. Am. 105(4), 2192-2218.

Bozorgnia, Y., N. A. Abrahamson, L. Al Atik, T. D. Ancheta, G. M. Atkinson, J. W. Baker, A. Baltay, D. M. Boore, K. W. Campbell, B. S. J. Chiou, R. Darragh, S. Day, J. Donahue, R. W. Graves, N. Gregor, T. Hanks, I. M. Idriss, R. Kamai, T. Kishida, A. Kottke, S. A. Mahin, S. Rezaeian, B. Rowshandel, E. Seyhan, S. Shahi, T. Shantz, W. Silva, P. Spudich, J. P. Stewart, J. WatsonLamprey, K. Woodell and R. R. Youngs (2014). NGA-West2 research project. Earthq. Spectra 30(3), 973-987. 
Campbell, K. W. (2003). Prediction of strong ground motion using the Hybrid Empirical Method and its use in the development of ground-motion (attenuation) relations in Eastern North America. Bull. Seismol. Soc. Am. 93(3), 1012-1033.

Campbell, K.W. and Y. Bozorgnia (2014). NGA-West2 ground motion model for the average horizontal components of PGA, PGV, and 5\% damped linear acceleration response spectra. Earthq. Spectra 30(3), 1087-1115.

Cauzzi, C., E. Faccioli, M. Vanini \& A. Bianchini (2015). Updated predictive equations for broadband (0.01-10s) horizontal response spectra and peak ground motions, based on a global dataset of digital acceleration records. Bull. Earthq. Eng. 13(6), 1587-1612.

Chiou, B. S. J. and R. R. Youngs (2008). An NGA model for the average horizontal component of peak ground motion and response spectra. Earthq. Spectra 24(1), 173-215.

Chiou, B. S. J., R. R. Youngs, N. A. Abrahamson and K. Addo (2010). Ground-motion attenuation model for small-to-moderate shallow crustal earthquakes in California and its implications on regionalization of ground-motion prediction models. Earthq. Spectra 26(4), 907-926.

Chiou, B. S. J. and R. R. Youngs (2014). Update of the Chiou and Youngs NGA model for the average horizontal component of peak ground motion and response spectra. Earthq. Spectra 30(3), 1117-1153.

Coppersmith, K. J., and R. R. Youngs (1986). Capturing uncertainty in probabilistic seismic hazard assessments within intraplate tectonic environments, Proc. 3rd US Nat. Conf. Earthq. Eng., vol. 1, 301-312.

Cotton, F., F. Scherbaum, J. J. Bommer, and H. Bungum (2006). Criteria for selecting and adjusting ground-motion models for specific target applications: applications to Central Europe and rock sites, J. Seismol. 10(2), 137-156.

Cremen, G., M. J. Werner and B. Baptie (2020). A new procedure for evaluating ground-motion models, with application to hydraulic-fracturing-induced seismicity in the United Kingdom. Bull. Seismol. Soc. Am., doi: 10.1785/0120190238.

Darzi, A., M. R. Zolfaghari, C. Cauzzi and D. Fäh (2019). An empirical ground-motion model for horizontal PGV, PGA, and 5\% damped elastic response spectra (0.01-10s) in Iran. Bull. Seismol. Soc. Am. 109(3), 1041-1057.

Delavaud, E., F. Scherbaum, N. Kuehn and C. Riggelsen (2009). Information-theoretic selection of ground-motion prediction equations for seismic hazard analysis: An applicability study using Californian data. Bull. Seismol. Soc. Am. 99(6), 3248-3263.

Donahue, J. L. and N. A. Abrahamson (2014). Simulation-based hanging wall effects. Earthq. Spectra 30(3), 1269-1284. 
Douglas, J., and B. Edwards (2016). Recent and future developments in earthquake ground motion estimation, Earth-Sci. Rev. 160, 203-219.

Douglas, J. and P. Jousset (2011). Modeling the difference in ground-motion magnitude-scaling in small and large earthquakes. Seismol. Res. Lett. 82(4), 504-508.

Douglas, J. (2019). Ground motion prediction equations 1964-2019. www.gmpe.org.uk

Farajpour, Z., S. Pezeshk and M. Zare (2019). A new empirical ground-motion model for Iran. Bull. Seismol. Soc. Am. 109(2), 732-744.

Fukushima, Y. (1996). Scaling relations for strong ground motion prediction models with $\mathrm{M}^{2}$ terms. Bull. Seismol. Soc. Am. 86(2), 329-336.

Gregor, N., N. A. Abrahamson, G. M. Atkinson, D. M. Boore, Y. Bozorgnia, K. W. Campbell, B. S.-J. Chiou, I. M. Idriss, R. Kamai, E. Seyhan, W. Silva, J. P. Stewart and R. Youngs (2014). Comparison of NGA-West2 GMPEs. Earthq. Spectra, 30(3), 1179-1197.

Hassani, B. and G.M. Atkinson (2018). Adjustable generic ground-motion prediction equation based on equivalent point-source simulations: Accounting for kappa effects. Bull. Seismol. Soc. Am. 108(2), 913-928.

Johnston, A. C. (1996). Seismic moment assessment of earthquakes in stable continental regions-I. Instrumental seismicity, Geophys. J. Int. 124(2), 381-414.

Kale, Ö. and S. Akkar (2013). A new procedure for selecting and ranking ground-motion prediction equations (GMPEs): The Euclidean distance-based ranking (EDR) method. Bull. Seismol. Soc. Am. 103(2A), 1069-1084.

Kotha, S.R., D. Bindi and F. Cotton (2016). Partially non-ergodic region specific GMPE for Europe and Middle-East. Bull. Earthq. Eng. 14(4), 1245-1263.

Kulkarni, R. B., R. R. Youngs, and K. J. Coppersmith (1984). Assessment of confidence intervals for results of seismic hazard analysis, Proc. $8^{\text {th }}$ World Conf. Earthq. Eng., San Francisco, vol.1, 263-270.

Miller, A. C. and T. R. Rice (1983). Discrete approximations of probability distributions. Manage. Sci. 29(3), 352-362.

PNNL (2014). Hanford Sitewide Probabilistic Seismic Hazard Analysis. PNNL-23361, Pacific Northwest National Laboratory, Richland, WA.

Rodriguez-Marek, A., E. M. Rathje, J. J. Bommer, F. Scherbaum, and P. J. Stafford (2014). Application of single-station sigma and site-response characterization in a probabilistic seismichazard analysis for a new nuclear site, Bull. Seismol. Soc. Am. 104(4), 1601-1619. 
Scherbaum, F., J. J. Bommer, H. Bungum, F. Cotton, and N. A. Abrahamson (2005). Composite ground-motion models and logic-trees: methodology, sensitivities and uncertainties, Bull. Seismol. Soc. Am. 95(5), 1575-1593.

Scherbaum, F., F. Cotton and P. Smit (2004). On the use of response spectral-reference data for the selection and ranking of ground-motion models for seismic-hazard analysis in regions of moderate seismicity: The case of rock motion. Bull. Seismol. Soc. Am. 94(6), 2164-2185.

Scherbaum, F., E. Delavaud and C. Riggelsen (2009). Model selection in seismic hazard analysis: An information-theoretic perspective. Bull. Seismol. Soc. Am. 99(6), 3234-3247.

Scherbaum, F., N. M. Kuehn, M. Ohrnberger and A. Koehler (2010). Exploring the proximity of ground-motion models using high-dimensional visualization techniques, Earthq. Spectra 26(4), 1117-1138.

Sedaghati, F. and S. Pezeshk (2017). Partially nonergodic empirical ground-motion models for predicting horizontal and vertical PGV, PGA and $5 \%$ damped linear acceleration response spectra using data from the Iranian plateau. Bull. Seismol. Soc. Am. 107(2), 934-948.

Stafford, P.J. (2014). Crossed and nested mixed-effects approaches for enhanced model development and removal of the ergodic assumption in empirical ground-motion models. Bull. Seismol. Soc. Am. 104(2), 702-719.

Stafford, P. J. (2015). Variability and uncertainty in empirical ground-motion prediction for hazard and risk analyses, Perspectives on European Earthquake Engineering and Seismology, vol. 2, $39,97-128$.

Stafford, P. J., F. O. Strasser and J. J. Bommer (2008). An evaluation of the applicability of the NGA models to ground-motion prediction in the Euro-Mediterranean region. Bull. Earthq. Eng. 6(2), 149-177.

Stafford, P.J., A. Rodriguez-Marek, B. Edwards, P.P. Kruiver and J.J. Bommer (2017). Scenario dependence of linear site-effect factors for short-period response spectral ordinates. Bull. Seismol. Soc. Am. 107(6), 2859-2872.

Stepp, J. C., I. Wong, J. Whitney, R. Quittemeyer, N. Abrahamson, G. Toro, R. Youngs, K. Coppersmith, J. Savy, T. Sullivan and Yucca Mountain PSHA Project Members (2001). Probabilistic seismic hazard analyses for ground motions and fault displacements at Yucca Mountain, Nevada, Earthq. Spectra 17(1), 113-151.

Stewart, J. P., J. Douglas, M. Javanbarg, Y. Bozorgnia, N. A. Abrahamson, D. M. Boore, K. W. Campbell, E. Delavaud, M. Erdik, and P. J. Stafford (2015). Selection of ground motion prediction equations for the global earthquake model, Earthq. Spectra 31(1), 19-45.

Strasser, F. O., N. A. Abrahamson and J. J. Bommer (2009). Sigma: Issues, insights, and challenges, Seismol. Res. Lett. 80(1), 40-56. 
Toro, G. R., N. A. Abrahamson, and J. F. Schneider (1997). Model of strong ground motions from earthquakes in central and eastern North America: best estimates and uncertainties, Seismol. Res. Lett. 68(1), 41-57.

USNRC (2018). Updated implementation guidelines for SSHAC hazard studies. NUREG-2213, US Nuclear Regulatory Commission Washington, D.C.

Yenier, E. and G. M. Atkinson (2015). Regionally adjustable generic ground-motion prediction equation based on equivalent point-source simulations: Application to central and eastern North America. Bull. Seismol. Soc. Am. 105(4), 1989-2009. 